thing useless must be wrong", and (2) that "there is no security problem in Nature", that is, Nature is no enemy striving to concoal the truth from us. He then attacked the view that the stable RNA of the ribosomes acts as a template and argued that the ribosomes are structures which synthesize, at a given time, the protein dictated by the attendant messenger. Thus he rejected two- and three-procoss models of transfer of information in favour of a one-process model which he claimed was universal.

The discussion was at first confined to thrust and counterthrust between the two experts, deftly provoked by the chairman. But gradually the audienco gained sufficient confidence to intervene. Questions about the evolution and the controlling mechanisms of this complex system (for example: How do you make a cat ?) led to lively discussions. It slowly became apparent that though genotic coding was concerned with transfer of information, information theory itself had little to offer in solving the problems of genetic coding apart from providing some descriptive terms. As Dr. Brennor put it in his final remark, "It's now just a matter of linguistics", that is, of reading the code.

The fourth and final discussion was devoted to philosophical aspects of cosmology. Prof. J. L. Mackie (Sydney) took the chair, and Mr. R. Harré (Oxford) and Dr. W. Davidson (Battersea College of Technology) were the two main speakers. Mr. Harré argued that tho universe, defined as 'everything there is', was logically not a proper subject for scientific study. He carefully distinguished between the cosmosciences and the astro-sciences by analysing the logical form of typical propositions of those subjects; though cosmography, he thought, could be regarded as scientific, the other cosmo-sciences, cosmogeny and cosmophysies, were merely spoculative. His main attack centred on the concept of infinity as used in or implied by the cosmo-sciences: he distinguished between the concept of an actual or referential infinity, which he rejected as logically improper, and the concept of a descriptive infinity which (analog. ously with the intuitionist concept of infinity in mathematics) has a finite starting point and a rule of progression which may continuo without limit and thus be a growing but potentially infinite sequence.

Dr. Davidson argued that development of the cosmo-sciences was scientifically both volid and feasible, and that such questions as whether the universe is spatially infinite or not, or whether it had an origin in time or not, are not necessarily transcendental issues. He claimed that the unity of science as a whole may depond on the development of a satisfactory cosmology in which every science could find its place.

With the two protagonists thus apparently diametrically opposed, the chairman formulated their main differences and members of the Conference set about their task of resolving them. In the discussion, Mr. Harré's dependence on Kantian antinomies, Russell's theory of types, and a priori arguments took some hard knocks. It scemed eventually that perhaps only the philosopher's concept of the universe was referentially infinite, while the cosmologist's univorse, if not finite, was at worst only descriptively infinite and therefore not, logically, a wholly improper subject of study.

The conference ended with a businoss meeting at which, inter alia, members were able to express their thanks to St. Hugh's College as hosts and to Dr. Mary Hesse as organizer for the parts they played in a pleasant and stimulating week-end.

B. C. Brookes

\title{
IONIC MELTS
}

T IQUIDS still constitute one of the least well 1 understood states of matter. Near the critical temperature various quasi-gaseous formulations of their behaviour are often quite successful, but near the melting point quasi-crystalline models offer more promise. Ionic melts have been much less studied than many other liquids. Their critical temperatures are usually not known, but are certain to be high. For this reason, current structural models for ionic melts show a bias towards quasi-crystalline concepts, which is strengthened by the strong statistical probability that each cation will have anions as its nearest neighbours, and vice versa, in order to lessen electrostatic repulsion energy.

However, really precise theories of the structure of ionic melts present difficulties which have not yet been surmounted. In addition to the theoretical difficulties, until recent years the amount and diversity of experimental work on ionic melts has been restricted by technical complications. Temperatures of study need to range from $100^{\circ}$ to well above $1,000^{\circ} \mathrm{C}$., and many of the melts attack practically every kind of containing wall. Despite these obstacles, interest in the science and technology of ionic melts has been growing rapidly. Sessions dealing with these systems have been included in a number of recent international scientific meetings, and two Gordon conferences in the United States have been wholly devoted to discussing their properties. At the discussion meeting of the Faraday Society, held in the University of Liverpool during September 5-7, seventy-five scientists from Britain and fifty from overseas had the opportunity of comparing progress in three main fields: structure, thermodynamic properties, and transport properties of ionic melts. The president, Sir Cyril Hinshelwood, was in the chair. Prof. H. Bloom, of the University of Tasmania, gave the eleventh Spiers Memorial Lecture on "Structural Models for Molten Salts and their Mixtures".

A broad conclusion from the discussion was that interesting and important oxperimental work is being carried out in quite a fow centres. Comprehensive theoretical treatments of the properties of ionic melts have by no means caught up with the range or the physical interest of the data now being obtained. Applied research on ionic melts in electrometallurgy or as heat-transfer fluids appears to be reasonably active in various countries. The number of substances capable of existence as stable liquids in the range $300-1,000^{\circ} \mathrm{C}$. is comparatively small, which points to the profitability of exploring further fields of application of ionic melts. At present, basic research on ionic melts seems scanty in British universities, 
having regard to the importance of extending physicochemical research to higher temperatures, and to the properties of condensed states of matter generally.

The first group of six papers presented at the Liverpool meeting of the Faraday Society were concerned with structure and spectra. In dealing with problems of structure, methods of describing and measuring the nearest neighbour and next-nearest neighbour environment of any ions continue to be of prime importance. Although they are indispensable, X-ray techniques applied to ionic melts suffer from the weakness that as with other liquids they only yield information in the form of radial distribution functions, which give average environments. In a significant number of ionic melts, there is evidence for a closer approach between anion and cation, and an increase of separation between ions of like sign, compared with the crystals. This shrinkage of contact distance between ions of unlike sign has been ex. plained in terms of the increased positional disorder in the melt, compared with the crystal. Disorder results in less complete compensation of electrostatic forces from the nearest neighbour shell of unlike ions, exerted on the ion at the centre of the shell. Greater local polarizations can be expected in the melt, even when the total volume it occupies is larger than when the same quantity of matter is in the crystalline state. Data were presented for radial distribution functions of molten carbonates and sulphates.

There was considerable discussion about the local arrangements of ions in close-packed melts. Spectroscopic techniques promise to give rather more specific information about local structure than is obtainable from X-ray determinations of radial distribution functions. Experimental studies presented for discussion included ultra-violet absorption spectra of transition metal ions in dilute solution melts. These yield information about ligand fields around the transition ion. A somewhat different type of ultraviolet absorption spectrum arises from so-called charge transfer effects, which involve excitation of an electron in the anion to an orbital the radius of which depends on that of the shell of cations immediately surrounding the anion. Such spectra can therefore yield valuable information about the configurational changes in this shell of nearest neighbours. One paper dealt with the modifications of charge transfer spectra that accompany various changes such as thermal expansion, the transition from one crystal form to another, and the transition from crystal to melt. Thermal expansions of crystalline nitrates observed by conventional means are found to match quite closely the expansions of the charge transfer orbitals as determined by the ultra-violet absorption spectra. This makes it all the more striking that volume changes on melting, as determined by conventional methods, are in the opposite direction to the changes in the charge transfer orbitals. Compared with the crystals, optical evidence for the melts thus points to a greater proportion of more tightly packed local assemblies of jons. Since the thermodynamic evidence shows an increase in volume, presumably this expansion results from increasing the proportion of vacancies and holes on melting alkali nitrates. Rubidium nitrate is one of the very few crystals which shrink on melting. Even here, the shrinkage of the charge transfer orbitals appears to be greater than the average contraction observed thermodynamically. For nitrates and other salts of polyatomic anions that have low melting points it is claimed that spectral evidence supports the general theory that the melting mechanism of these crystals includes the formation of 'association complexes', as well as the more usual increase of positional disorder and randomization of orientation of non-spherical ions. A second paper on charge transfer spectra described changes in mixed molten nitrates in terms of the effective electrostatic fields due to neighbours and next-nearest neighbours. Interesting information about the structure of liquids seems likely to emerge from extensions of such studies on mixtures of molten salts.

There was considerable but not very conclusive discussion about the usefulness or otherwise of attempting experimental and even conceptual distinction between 'association complexes' and 'complex ions'. It seems to be generally agreed that complex ions can be readily distinguished when they are formed in dilute solution in ionizing solvents; many techniques for their identification have been developed since Arrhenius. In condensed ionic assemblies, whether crystalline or molten, the distorting influence of nearest neighbours can make identification of a complex much less discriminating. 'Association complexes' can be the result of various ways of packing ions so as to minimize their potential energy. In discussing melting mechanisms, it is important to recognize that some of these packings are 'non-crystallizable'; for these, addition of more and more ions according to the same rules of packing will not fill space economically. Only crystal nuclei can be extended by packing additional ions indefinitely in three directions in space; but so long as they are quite small, non-crystallizable association complexes may compete thermodynamically with potential crystal nuclei in the melt, which can be regarded as containing a mixture of the various kinds.

For discussing melting mechanisms, it may not be necessary to decide whether the reduction of potential energy on passing from random ionic packing to an association complex results primarily from electrostatic effects, or whether recognizable 'complex ions' are formed in which delocalization of valency electrons may extend over all the atoms in the complex.

But for the interpretation of other properties of ionic melts, the identification of strongly bonded complex ions may be more urgent. For example, cryoscopic measurements continue to yield suggestive structural information. One group of studies presented for discussion referred to the formation of complex ions such as $\mathrm{CdCl}_{3}$ or $\mathrm{PbCl}_{3}$ - analogous to those encountered in aqueous ionic solutions.

A novel field is opened up by cryoscopic and other studies of the behaviour of solutions of metals in ionic melts. In some of these solutions the electron appears to leave the metal atom and may have a role analogous to that in solutions of alkali metals in liquid ammonia. Theoretical and experimental aspects of the electronic properties of such solutions received considerable discussion. Transport of electrons under the influence of electric fields applied to such systems has interesting contrasts and analogies with the movement of eharge earriers in semiconductors. Conductances can be quite high even when the concentration of the metal atom is fairly dilute. Developments of these investigations promise to throw much additional light on conductance mechanisms generally that do not involve ion migration in condensed phases.

Papers presented at the meeting point to significant advances in the precision of measurement of basic 
thermodynamic parameters of ionic melts, such as activity coefficients. Evaluations were reported of excess functions for the enthalpy and changes in volume on mixing ionic melts of the same or of different valency structure. As a result, some of the current quantitative theories that have been developed for non-ionic liquid mixtures can begin to be adapted to ionic melts. Considerable advances may be expected in the next few years in this branch of the theory of liquids.

One-third of the papers presented dealt with transport properties of ionic melts, which have been studied for a number of years and which continue to receive much attention. Use of radioisotopes has greatly enriched the possibilities of making measurements on diffusion, the correlation of which with other transport phenomena helps to unravel complicated structural problems. In addition to their technological importance, transport properties can yield tests of theoretical models for ionic melts that can be much more sensitive than equilibrium properties to structural details assumed in the model. Discussion of measurements of thermo-electric power of ionic melts suggested further interesting possibilities that seem likely to attract additional attention in the years ahead.

A. R. UвBELOHDE

\section{WOOD EXTRACTIVES}

$\mathrm{A}$ SYMPOSIUM on wood extractives was arranged by the Plant Phenolics Group and held at the Royal Holloway College, Englefield Green, Surrey, during September 20-22. The meeting proved valuable in bringing together workers in different fields, including several from overseas, having a common interest in the minor or extraneous components of woods. After an introductory session the subject of wood extractives was dealt with under three headings, namely biosynthesis and taxonomy, the chemistry of extractives, and their influence on utilization of wood and wood products.

Dr. F. E. King (British Petroleum Co., Itd.) opened the symposium with an introductory address entitled "Perspectives in the Chemistry of Wood Extractives". Dr. King surveyed the historical development of investigations on extractives, commencing with those of technical interest such as the tannins, dyestuffs and resins, and proceeding to the work of Erdtman on the heartwood components of coniferous trees and his own work on the corresponding components of hardwoods. These investigations led to the conception of heartwood components as 'taxonomic tracers' which can be used to supplement the observations on morphological features employed by botanists in the taxonomic classification of trees. Dr. King emphasized the wide variety of $\mathrm{C}_{6}-\mathrm{C}_{3}-\mathrm{C}_{6}$ compounds that are found in woods and discussed a fow unusual structures that have been encountered.

In the second paper, given by Prof. F. W. Jane (Royal Holloway College), an account was given of the structure of the tree and the distribution of the various tissues in it. This was helpful to the audience, consisting predominantly of chemists. The extraneous substances are classified by botanists as gums, resins, tannins, oils, mucilages, colouring matters, mineral deposits, etc., and the location of these in the structure was described and illustrated. There is clearly room for closer co-ordination between the observations of botanists, based largely on the microscopic staining reactions of these components, and their investigation by chemists.

Prof. E. J. Bourne (Royal Holloway College) described some new methods for the characterization and separation of extractives that have been studied in his laboratory. These include the use of molybdates and tungstates which form com. plexes with polyhydroxy compounds and can assist in their separation by electrophoresis, the addition of phenylboronic acid to the solvents used in paper chromatographic separations of these com- pounds, the use of boron trichloride under very mild conditions to identify families of extractives by removing substituent groups, and the fractionation of mixtures by means of a new liquid/liquid countercurrent extraction technique, which was demonstrated.

The session on biosynthesis and taxonomy opened with a paper by Prof. W. Sandermann (Bundesforschungsanstalt für Forst- und Holzwirtschaft, Reinbek, West Germany) on the biogenesis of terpenes in plants. Prof. Sandermann discussed the part played by mevalonic acid and its conversion into geranyl and farnesyl pyrophosphates, from which the more complex terpenes may be derived. He then described his work on the incorporation of labelled precursors in plants and showed how a distinction between alternative mechanisms can be made by these methods; for example, the biosynthesis of $\alpha$-pinene and thujone was shown to take place by an electrophilic substitution rather than by an addition mechanism. A series of terpenoid compounds has been found in teak and correlated structurally with the anthraquinones present in this wood.

The systematic distribution of phenolic substances in plants was discussed by Dr. E. C. Bate-Smith and Dr. T. Swain (Low Temperature Research Station, Cambridge) in a paper presented by Dr. Swain. The contribution that a study of the chemical components of plants can make to taxonomic classification was emphasized. One of the results of an extensive survey of the occurrence of the common phenolics is the recognition that plants in some families or higher groupings are devoid of the capability of synthesizing certain types of compound. In this connexion the occurrence of compounds containing vicinal trihydroxy groups and of ellagic acid in members of the Rosaceae was discussed.

In continuation of this theme Prof. C. W. L. Bevan (University College, Ibadan, Nigeria) presented a paper entitled "Wood Extractives as Tracer Substances in Taxonomy", and referred particularly to the results of a survey carried out in his Depart. ment of components of woods of the family Meliaceae. He also outlined the procedures followed in determining the structures of gedunin, isolated from Entandrophragma angolense and of khivorin from Khaya ivorensis and $K$. grandifoliola.

In the session on the chemistry of wood extractives four papers were presented, dealing respectively with the lignans, tropolones, stilbenes, and flavonoids, these being the principal types of phenolic compounds 